\title{
Evaluation of Morphological Effect on Thermal and Mechanical Performance of PS/PMMA/CdS Nanocomposite Systems
}

\author{
Vishal Mathur ${ }^{1,2,3}$, Kananbala Sharma ${ }^{3}$ \\ ${ }^{1}$ Department of Physics, S. S. Jain Subodh P.G. College, Jaipur, India \\ ${ }^{2}$ Department of Engineering Physics, Kautilya Institute of Technology \& Engineering, \\ Riico Institutional Area Sitapura, Jaipur, India \\ ${ }^{3}$ Semiconductor and Polymer Science Laboratory, Vigyan Bhawan, University of Rajasthan, Jaipur, India \\ Email: wishalmathur@gmail.com,wishalmathur@yahoo.co.in
}

Received March 6, 2013; revised April 6, 2013; accepted April 15, 2013

Copyright (C) 2013 Vishal Mathur, Kananbala Sharma. This is an open access article distributed under the Creative Commons Attribution License, which permits unrestricted use, distribution, and reproduction in any medium, provided the original work is properly cited.

\begin{abstract}
In the present paper an effort has been made to investigate effect of dispersion of CdS nanoparticles on the thermal and mechanical properties of PS/PMMA blends. Samples have been prepared through dispersion of CdS nanoparticles (prepared separately) during solution casting blend fabrication processing. These nanocomposites samples are structurally characterized through Wide angle X-ray Scattering (WAXS) and Small Angle X-ray Scattering (SAXS) techniques. Scanning Electron Microscopy (SEM) analyses of these samples have been carried out in lieu of surface morphological characterization. The measurements of glass transition temperature and stress-strain analyses have been performed through Dynamic Mechanical Analyzer (DMA). The thermal conductivity of nanocomposite samples has been determined using Hot Disk Thermal Constants Analyzer. The study shows that the incorporation of dispersed CdS nanoparticles in PS/PMMA blend matrix significantly alter their glass transition behaviour, thermal conductivity and tensile properties.
\end{abstract}

Keywords: Thermal Conductivity; CdS Nanocomposites; Glass Transition Temperature; Tensile Properties

\section{Introduction}

High performance in polymer blends and composites can be achieved through the addition of a strong filler component into a polymer matrix. In this regard polymer nanocomposites present a promising alternative of conventional composites. Recently, studies on blends of immiscible polymers containing nanoparticles have attracted the attention of several research groups [1-5]. Chung et al. [6] made the first quantitative study of phase separation dynamics in a polymer blend containing mobile 22 $\mathrm{nm}$ silica nanoparticles. These studies suggest that under certain conditions the polymer molecules and the nanoparticles should not be regarded as individual entities within the blend, but instead as complex aggregates. Numerical simulations of polymer nanocomposites have shown that the introduction of hard spheres into a polymer blend has similar effects to the introduction of new polymer/hardwall interfaces [7,8]. Vacatello [9] performed simulations of polymer systems filled with particles of a size comparable to the polymer chains and found that even in the absence of specific interactions with the polymer, the filler particles behaved as highly functional physical crosslinks, reducing the overall mobility of the polymer chains compared to the unfilled polymer matrix.

Nanoparticles particularly II-VI semiconductors have attracted wide spread attention, because they are relatively easy to synthesize in the size range required for the quantum confinement. In accordance with the need, various inorganic semiconductors such as CdSe, CdTe, CdS, and $\mathrm{PbS}$, have been actively investigated and among these CdS has been known as one of the most promising photo-sensitive material owing to its unique photochemical activities and strong visible-light absorption and emission property [10]. Hence, it has many commercial or potential applications in light-dependent resistors, solar cells, or other photo-electronic devices [11-13]. The nanocomposite of CdS can provide the possibility to provide combinations of functionalities, such as thermally conducting composites with good mechanical properties that are optically clear. Such properties can result because 
CdS nanoparticles, with diameters distinctly below the Rayleigh scattering limit, still display their solid-state physical properties when embedded in transparent matrices. Basically CdS nanocomposites are optical composites and most of the studies of these are concerning optical characterization [14-17]. The different technological applications of CdS nanocomposites include biological labeling and diagnostics, LED's, electro-luminescent devices, photovoltaic devices, lasers and single electrode transistors. These applications of CdS nanocomposites in various fields necessitate the proper understanding of their thermal and mechanical properties because along with mechanical performance, the system has to entail heat transition phenomenon almost in all applications.

In view of this, the present work is aimed to establish concepts to obtain nanocomposites with desired properties to enhance compatibility of immiscible blends as well as to understand the mechanical and thermal stability of these systems. An extensive study of thermal and mechanical properties through two major experimental set up namely Hot Disc Thermal Constant Analyzer (TPS) and Dynamic Mechanical Analyzer (Tritec 2000 DMA), respectively have been under taken in detail. In view of this, above mentioned aspects have been studied using PS/PMMA blends and their CdS nanocomposites (PS/ PMMA/CdS). PS and PMMA both are well known due to their various commercial and domestic applications and stand as primary industrial polymeric materials $[18$, 19]. The study shows that the incorporation of dispersed CdS nanoparticles in PS/PMMA blend matrix significantly alter their glass transition behaviour, thermal conductivity and tensile properties.

\section{Experimental}

\subsection{Material Preparation}

In order to prepare polymeric blended-nanocomposite samples, firstly $\mathrm{CdS}$ nanoparticles have been prepared by simple chemical method using $\mathrm{CdCl}_{2}$ and $\mathrm{H}_{2} \mathrm{~S}$ gas produced from thiourea [20]. The samples of PS/PMMA/CdS polymeric nanocomposite have been prepared by dispersing prepared $\mathrm{CdS}$ nanoparticles during the preparation of polymeric blends of different concentrations $(0 \%, 30 \%$, $50 \%, 70 \%$, and $100 \%$ ) of PS/PMMA through solution casting method. In this method the laboratory grade polymeric material which are to be blend are dissolved accordingly in the tetrahydrofurane (T.H.F.) solvent. Then $10 \%$ chalcogenide CdS nanofiller particles of blending composition were dispersed in this solution. This solution was then stirred with the help of magnetic stirrer and then poured into flat-bottomed petri dishes to form film with a thickness of $\sim 0.05 \mathrm{~mm}$. The solvent is allowed to evaporate slowly over a period of 24 hours in dry atmosphere. The so obtained film was then peeled off and dried in vacuum at $50^{\circ} \mathrm{C}$, well below the boiling point of solvent to avoid bubbling, for 24 hours in order to ensure the removal of the solvent [21,22].

\subsection{Hot Disk Thermal Constants Analyzer Measurements}

Thermal transport properties of PS/PMMA polymeric blends of thickness about $2 \mathrm{~mm}$ have been measured through the Hot Disk Thermal Constants Analyzer from room temperature to $120^{\circ} \mathrm{C}$. It is based on transient plane source (TPS) technique. The TPS technique consists of an electrically conducting pattern, in the form of a bifilar spiral, which serves both as the source of heat and as a temperature sensor. The sensor is sandwiched between the thin insulating layers of Kapton. The TPS sensor element is made of $10 \mu \mathrm{m}$ thick nickel foil with an insulating layer made of $50 \mu$ m-thick Kapton, on each side of the metal pattern. The voltage increase over the sensor is recorded precisely. The evaluation and measurements of thermal conductivity and thermal diffusivity were performed in a way mentioned by Gustafsson [23] and computer software supplied in TPS $2500 \mathrm{~S}$.

\subsection{Dynamic Mechanical Analyzer (DMA) Measurements}

Dynamic Mechanical Analyzer (TRITEC-2000 DMA) is a sensitive technique that characterizes the mechanical response of materials by monitoring property change with respect to the temperature and frequency of applied sinusoidal stress. DMA film samples were cut to be between $4-6 \mathrm{~mm}$ in width and $10 \mathrm{~mm}$ in length. The average thickness of each sample is of 100-micrometer order. After adjusting DMA device in tension mode, the furnace was sealed off, sample scanned over a temperature range from room temperature to $140^{\circ} \mathrm{C}$. The sample was held at that temperature for five minutes. The heating/ramp rate was $2^{\circ} \mathrm{C} / \mathrm{min}$ for all temperature scan tests. Frequency of oscillations was fixed at $1 \mathrm{~Hz}$ and strain amplitude $0.01 \mathrm{~mm}$ within the linear visco-elastic region. The storage modulus E', loss modulus E" and mechanical loss factor ( $\operatorname{Tan} \delta$ ) have been determined during the test as a function of increasing temperature [22,24].

\section{Results and Discussion}

\subsection{Structural and Surface Morphological Characterization}

The structural characterization of PS/PMMA/CdS nanocomposites is primarily concerned with investigating whether the dispersed CdS nanoparticles are still within nano dimension. Two major techniques, namely, wide angle Xray scattering (WAXS) \& small angle X-ray scattering (SAXS), were used to ascertain the nano dimen- 
sion such. Surface morphological characterizations of the PS/PMMA/CdS nanocomposites were done through scanning electron microscope (SEM) analysis.

\subsubsection{Wide Angle X-Ray Scattering Analysis}

WAXS is used to estimate the particle size of prepared $\mathrm{CdS}$ nanoparticles. Figure 1 shows the WAXS pattern of
CdS nanoparticles. The presence of broad peaks confirms the nano dimension of the prepared nanoparticles. The average particle size obtained from the Debye Scherrerr formula [20] is $3 \mathrm{~nm}$. The WAXS patterns for the 50PS/ 50PMMA \& 50PS/50PMMA/CdS nanocomposite is as shown in Figure 2.

A broad hallow in the $2 \theta$ range of $25^{\circ}-40^{\circ}$ is observed

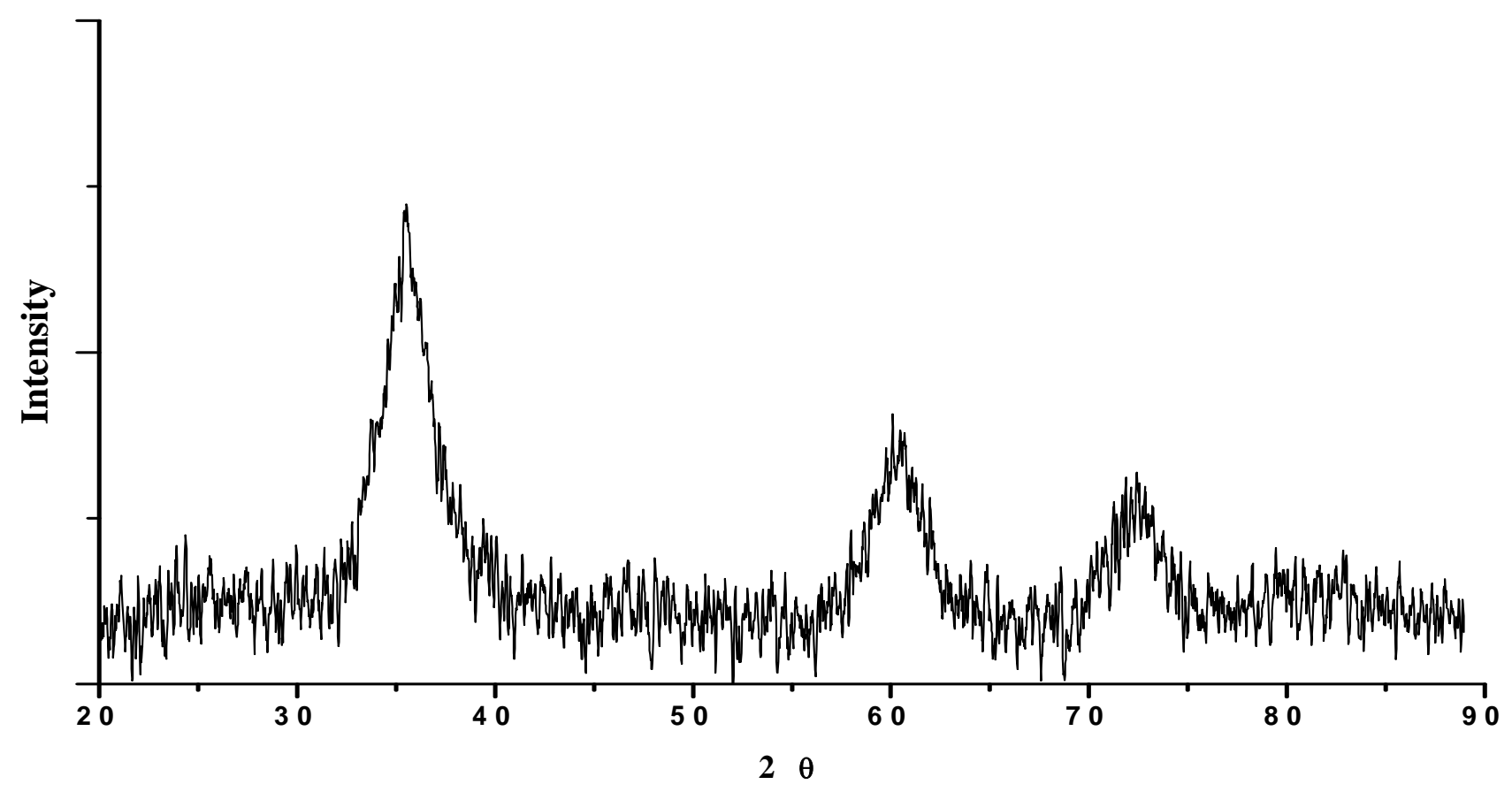

Figure 1. WAXS pattern of CdS nanoparticles.

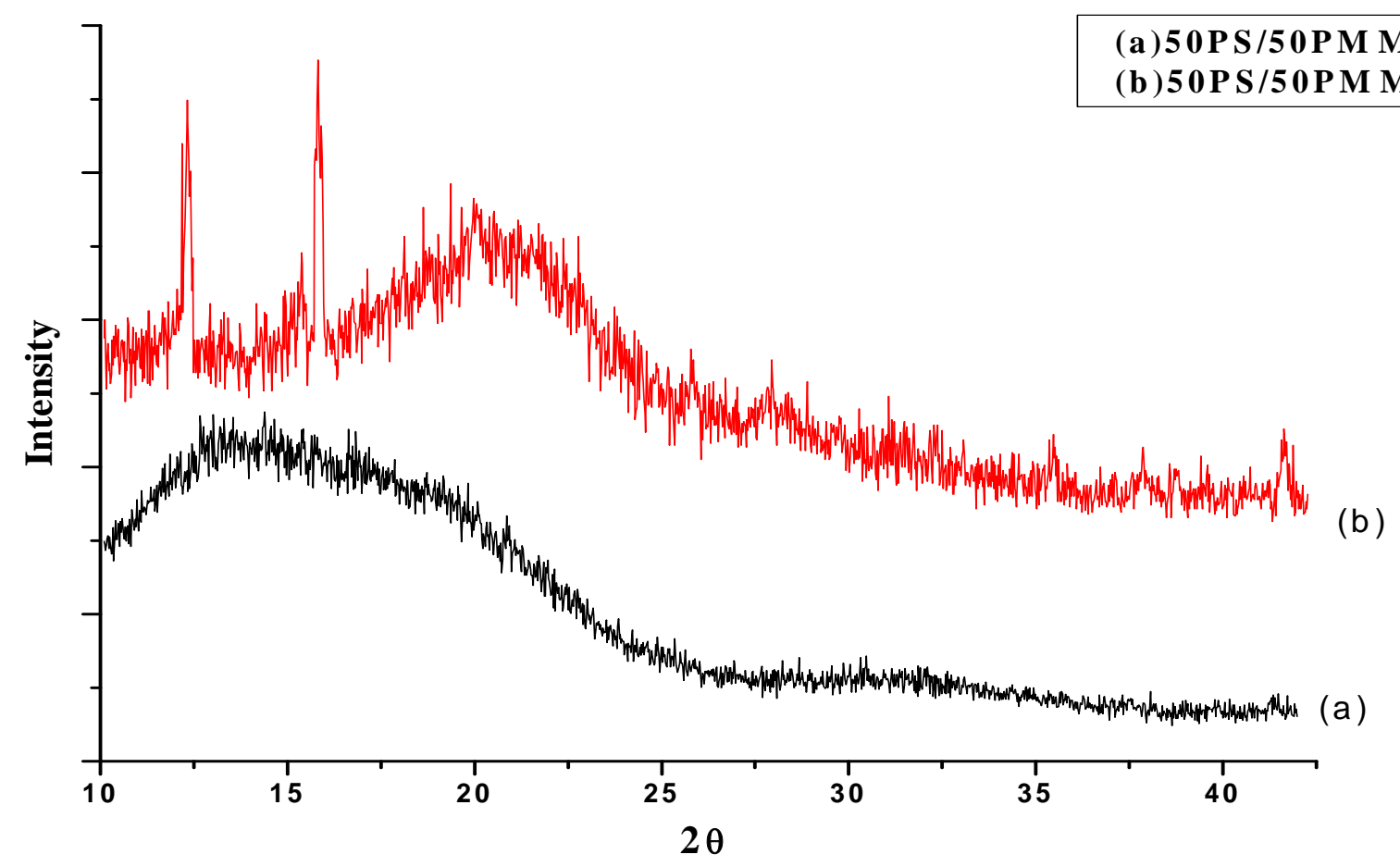

Figure 2. WAXS pattern of 50PS/50PMMA \& 50PS/50PMMA/CdS. 
in the WAXS pattern for 50PS/50PMMA and this is characteristic of the polymer. The pattern for the 50PS/ 50PMMA/CdS nanocomposite exhibits a semi-crystalline nature. It can be seen that the random dispersion of CdS nanoparticles within the 50PS/50PMMA matrix provides high scattering centers for the incident X-rays giving intense diffraction peaks. The peak observed in the pattern of the nanocomposite at $12^{\circ}$ and $17^{\circ}$ is thus also a consequence of this type of scattering of X-rays by the $\mathrm{CdS}$ nanofiller. Similar observations have also been reported by other researchers $[25,26]$. The pattern for nanocomposite sample does not yield information about the distribution of nanoparticles within the sample. To ascertain the above perspectives small angle X-ray scattering analysis is used.

\subsubsection{Small Angle X-Ray Scattering Analysis}

SAXS measurements were performed on an X'Pert Pro MPD system to investigate the size of the CdS-nanoparticles within nanocomposite samples. For the determination of size, shape, and distribution of particles, data files obtained from the X'Pert Pro MPD system were matched with the analysis templates that are provided with the EasySAXS software. Figures 3(a)-(c) show the scattering intensity as a function of angle $(2 \theta)$ for PS, PMMA, 50PS/50PMMA \& their CdS nanocomposites respectively.

It is observed that SAXS pattern of CdS embedded polymer nanocomposites show higher scattering intensity as compared to without $\mathrm{CdS}$ dispersed samples. The nanocrystals of $\mathrm{CdS}$ act as independent scattering centers in the respective polymer matrices and add to the total scattering intensity in the respective SAXS pattern. SAXS patterns are used for elucidating the shape and size of $\mathrm{CdS}$ nano-crystals by subtracting the background scattering intensity (without CdS embedded polymeric phase) from scattering intensity of CdS embedded polymeric/ nanocomposite phases with the help of EasySAXS software. SAXS characterization reports of respective nanocomposites suggest that $\mathrm{CdS}$ nanofiller particles have been distributed evenly within the available polymer/ blend matrix and have very little tendency to form agglomerates.

Figure 4 shows the particle size distribution curves for the PS/CdS, PMMA/CdS and 50PS/50PMMA/CdS nanocomposites. The particle size distribution report of this nanocomposite suggests that the distribution is well approximated by a Gaussian. The minor oscillations in the distribution curve around zero toward larger particle radii may be regarded as insignificant. It is observed that particle size distribution curve for PS/CdS, \& PMMA/CdS nanocomposites respectively, are approximately centered at $\mathrm{R}=1.3 \mathrm{~nm}$ and it means that the most frequent radius (R) of CdS nanofillers is $1.3 \mathrm{~nm}$ and major volume frac- tion of these CdS nanoparticles is exhibiting radius within $0.6 \mathrm{~nm}$ to $2.6 \mathrm{~nm}$. Similarly from curve (c), it is revealed that $\mathrm{R}=3.5 \mathrm{~nm}$ is as a most frequent radius of CdS nanofillers along with their particle size variation from $\mathrm{R}=$ $6.8 \mathrm{~nm}$ to $8.8 \mathrm{~nm}$ within the 50PS/50PMMA/CdS nanocomposite matrix. Hence the observed values of average particle size of $\mathrm{CdS}$ nanoparticles in all respective nanocomposite samples lies within nano dimensions (i.e. below $100 \mathrm{~nm}$ ) and implies that the prepared samples retain their nanocomposite nature.

\subsubsection{Scanning Electron Microscopy Analysis}

The realistic picture of the degree of $\mathrm{CdS}$ nanofillers dispersion in the nanocomposites can be obtained through scanning electron microscope analysis. Figure 5 shows the cross-section SEM micrographs of the PS, PMMA, 70PS/30PMMA, 50PS/50PMMA and 30PS/70PMMA blends with and without $\mathrm{CdS}$ nanoparticles. It can be seen that the CdS nanoparticles have been uniformly dispersed in each polymer base matrix but in dissimilar manner, respectively. In $\mathrm{PS} / \mathrm{CdS}$, the concentration of $\mathrm{CdS}$ nanoparticles on the exterior surfaces of PS phase makes possible large number of contacts and nanophase separation is observed in this nanocomposite.In PMMA/CdS, CdS nanoparticles settle down at the void sites of PMMA matrix and form exfoliated nanocomposite. SEM image of PS/CdS showed some level of flocculation of $\mathrm{CdS}$ nanoparticles, but of varying sizes. But in the PMMA/ $\mathrm{CdS}$ nanocomposite, CdS flocculants were limited and improvement in compatibility of this system have been observed.

The immiscible interphases of PS and PMMA exhibited in SEM images of PS/PMMA, are not observed in SEM images of PS/PMMA/CdS nanocomposites. It suggests that the overall compatibility of the respective blend system increases. The effect of different morphological structures of respective nanocomposite is further reflected in different bulk properties offered by them.

\subsection{Glass Transition Temperature Measurements}

The knowledge of glass transition temperature $\left(T_{g}\right)$ is one of the benchmark used to compare the thermal behavior of composites. Changes in the $\mathrm{T}_{\mathrm{g}}$ as a function of the filler content have been reported [27-31] for polymer composites containing a wide variety of fillers and polymers. Results of investigations of PS/PMMA/CdS nanocomposites are shown in Figures 6(a)-(e), respectively. These figures depict the variation of the Tan $\delta$ of the nanocomposite samples as a function of temperature.

It has also been observed that $\mathrm{T}_{\mathrm{g}}$ of $\mathrm{CdS}$ embedded PMMA phase shifts towards the higher temperature side with the increase of PS content whereas $\mathrm{T}_{\mathrm{g}}$ of $\mathrm{CdS}$ em- 


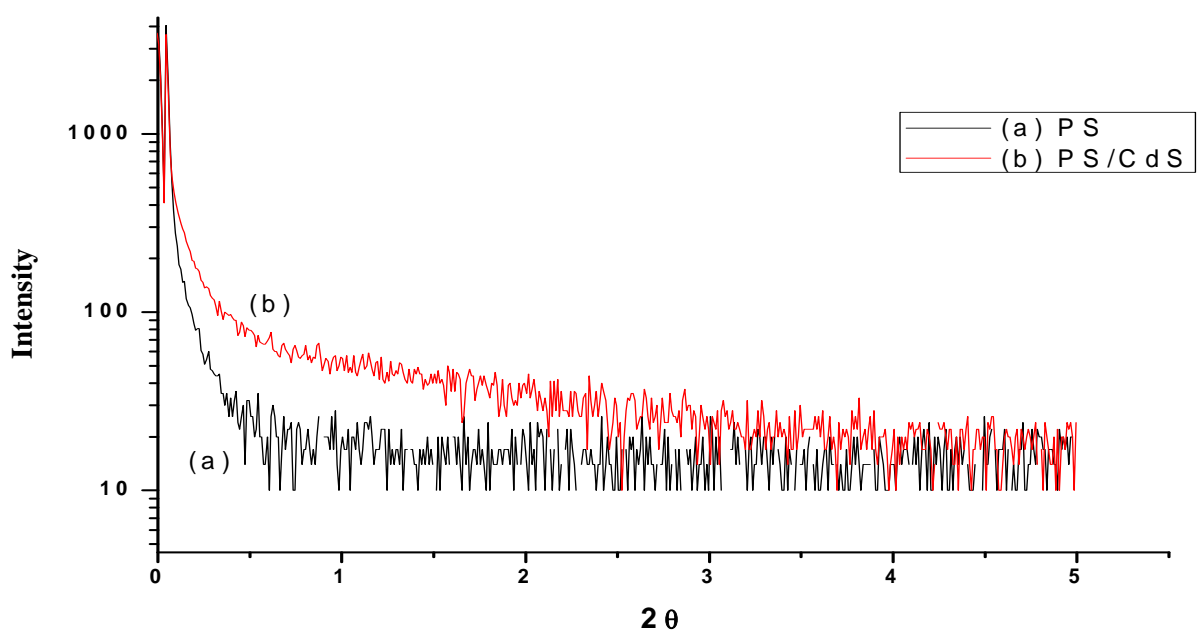

(a)

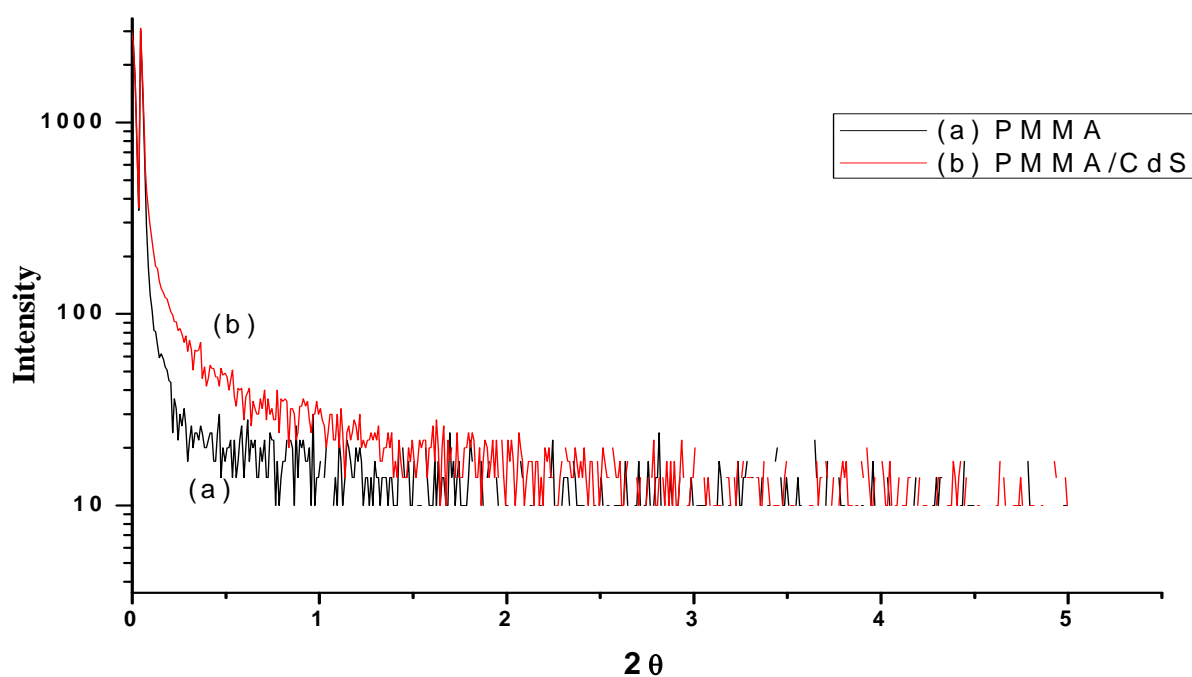

(b)

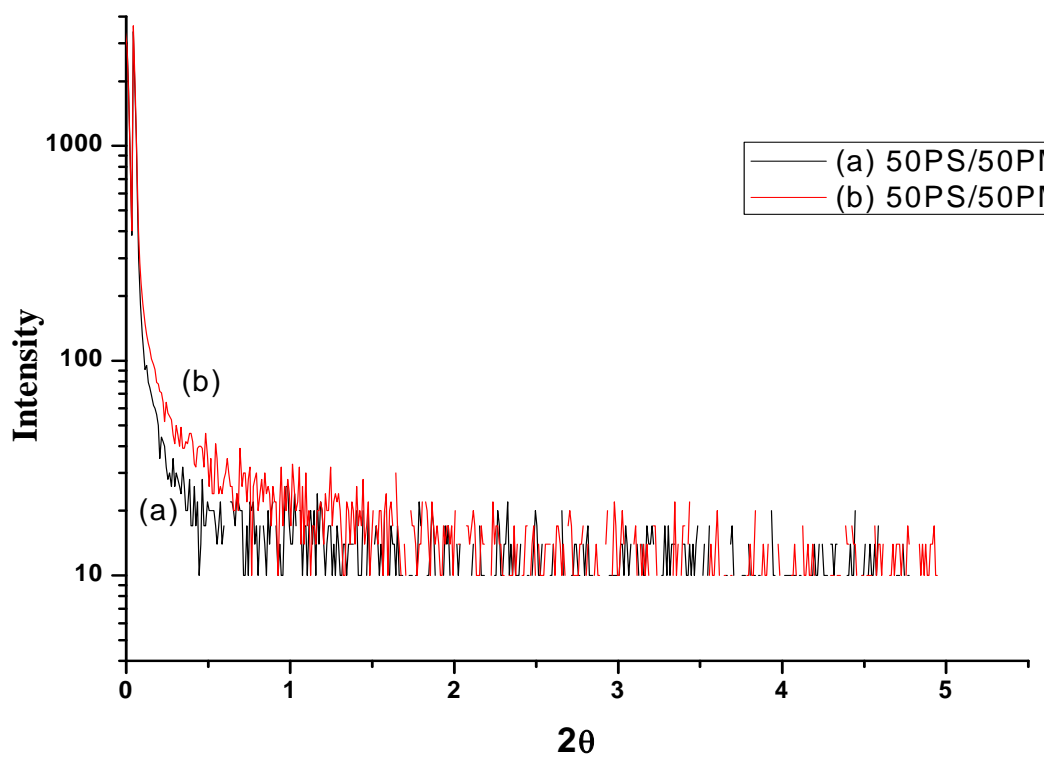

(c)

Figure 3. SAXS pattern of CdS nanocomposites of PS, PMMA \& 50PS/50PMMA. 


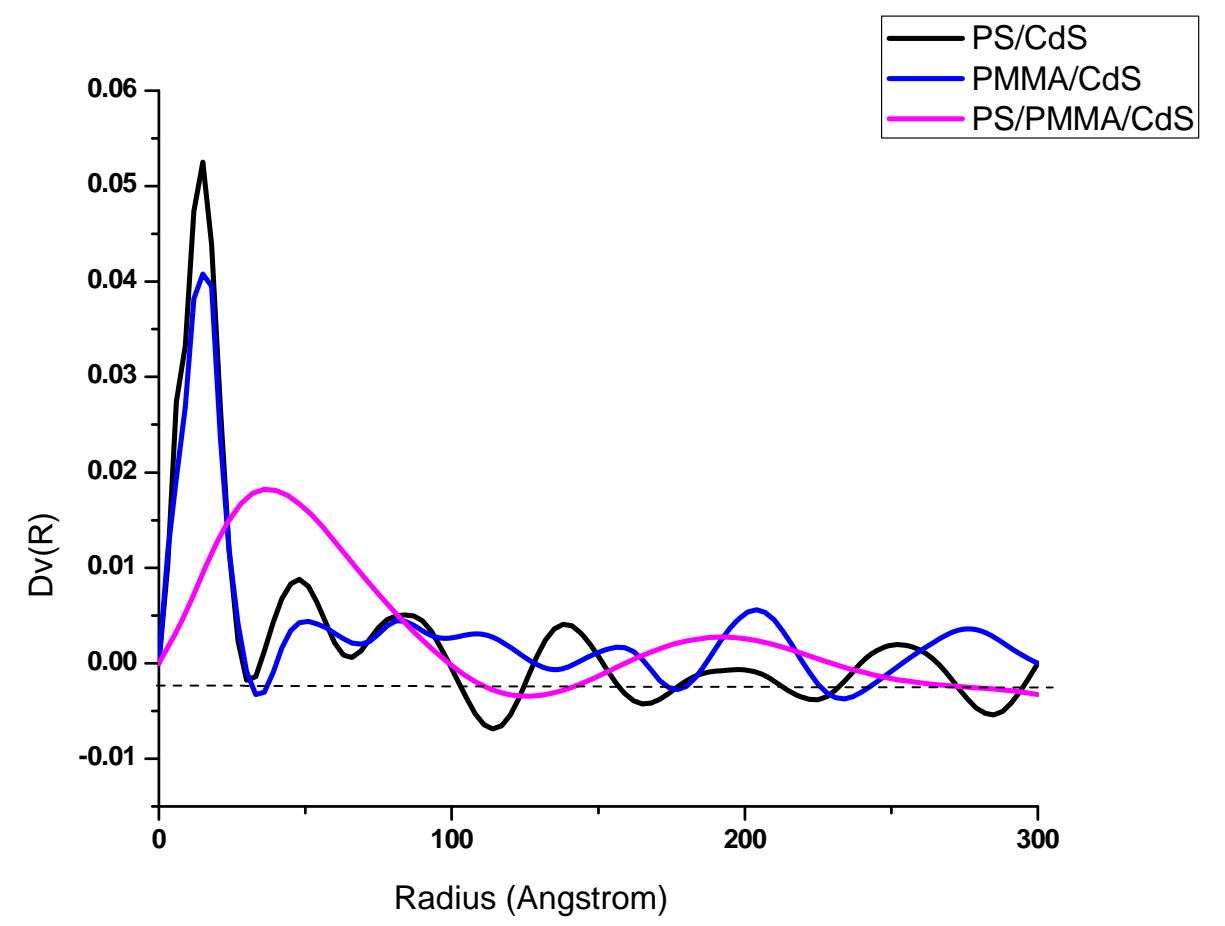

Figure 4. Particle size distribution curves for PS/CdS, PMMA/CdS \& 50PS/50PMMA/CdS.

bedded PS phase seems to be statistically constant in all $\mathrm{PS} / \mathrm{PMMA} / \mathrm{CdS}$ nanocomposite samples. The $\mathrm{T}_{\mathrm{g}}$ values corresponding to PS/PMMA blends \& PS/PMMA/CdS nanocomposite samples are tabulated in Table $\mathbf{1}$.

This study has demonstrated that in polymer blend/ nanocomposite samples, glass transition temperatures of corresponding phases are significantly improved. This effect is similar to those reported in the work of Chung et al. [6]. According to their reported work, change in the $\mathrm{T}_{\mathrm{g}}$ is attributed to the change in the viscosity of the PS phase. The enrichment of nanoparticles at interfaces between the polymeric blend phases has also been found in numerical simulations $[1,2,7,8]$ that indicate that there may be an accumulation of the nanoparticles at the phase interfaces with similar energetic interaction of nanoparticles for both polymer phases. In the present study it appears that this energetic interaction between respective polymer phases and nanoparticles is of van der waal type interaction that restricts the mobility of polymer chains and thus more energy is required by the system to achieve the glass transition state and therefore an increase in glass transition temperature of that particular polymeric phase in respective nanocomposite series, is observed.

\subsection{Thermal Conductivity Measurements}

Thermal conductivity of PS/PMMA blends and their CdS nanocomposite thin film samples (thickness $\approx 0.1 \mathrm{~mm}$ ) have been determined through transient plane source technique using thin film method with an input power of 0.5
$\mathrm{W}$ for 5 seconds at room temperature $(303 \mathrm{~K})$. Figure 7 shows the thermal conductivities of PS/PMMA/CdS nanocomposites as a function of weight $\%$ composition of PMMA in PS matrix, respectively.

The values of thermal conductivities obtained for PS/ PMMA blends and their CdS nanocomposites are summarized in Table 2.

The first observation is that the thermal conductivities of studied CdS nanocomposite samples are higher than their counterparts without $\mathrm{CdS}$ dispersed respective samples. As we know in non-metallic material system like polymer and polymer blends, the thermal energy is mainly carried by phonons, which have a wide variation in frequency and the mean free paths. The heat carrying phonons often have large wave vectors and mean free paths in the order of nanometer range at room temperature. In this way the dimensions of the nanoparticles become comparable to the mean free paths which would lead to significant improvement in phonon transport within the nanocomposite materials. The phonon confinement and quantization of phonon transport results modification in thermal properties. Since CdS nanoparticles having higher thermal conductivity and act as cross-linking centers between the polymer molecules therefore promptly subserve for the phonon-phonon conduction in that respective polymer network. This causes the enhancement of thermal conduction in $\mathrm{CdS}$ nanocomposites of PS/ PMMA blends.

Besides this, interfaces included in nanocomposite samples are also very important factor for consideration in 


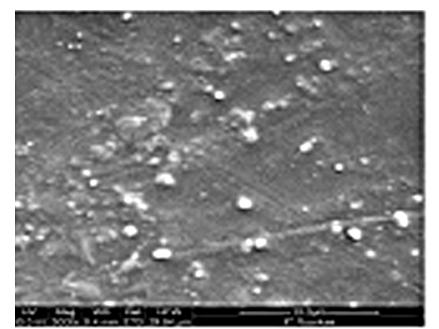

(a)

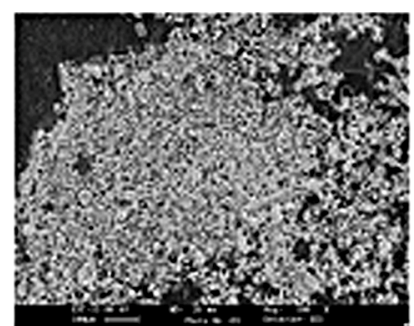

(b)

SEM micrographs of (a) PS (b) PS/CdS

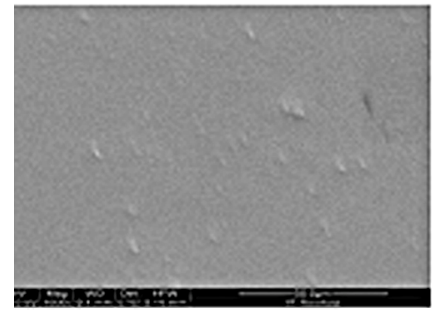

(a)

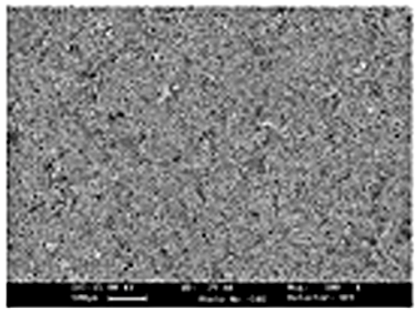

(b)

SEM micrographs of (a) PMMA (b) PMMA/CdS

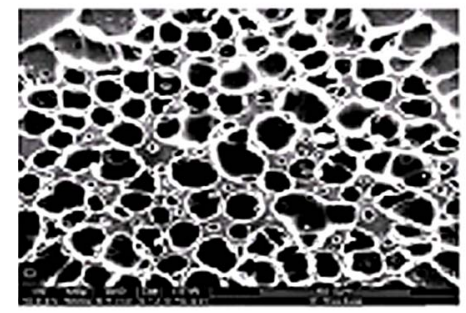

(a)

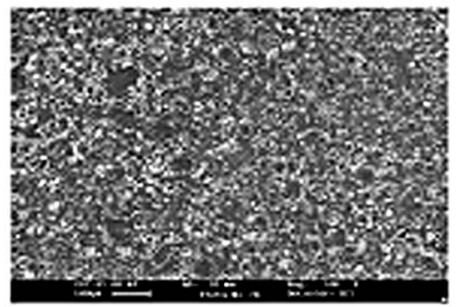

(b)

SEM micrographs of (a) 70PS/30PMMA (b) 70PS/30PMMA/CdS

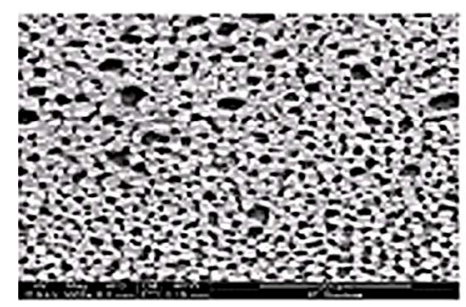

(a)

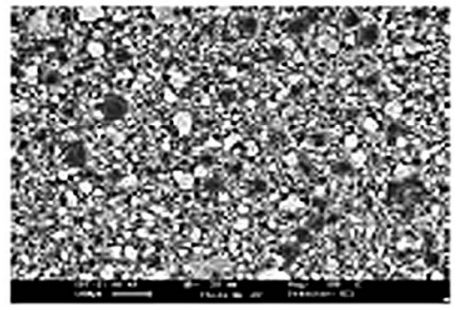

(b)

SEM micrographs of (a) 50PS/50PMMA (b) 50PS/50PMMA/CdS

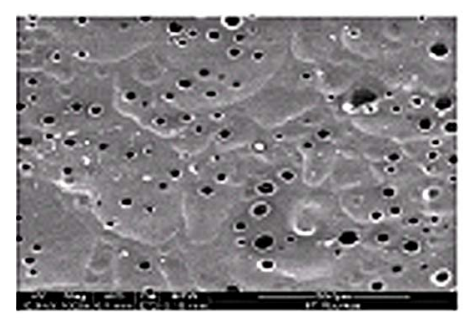

(a)

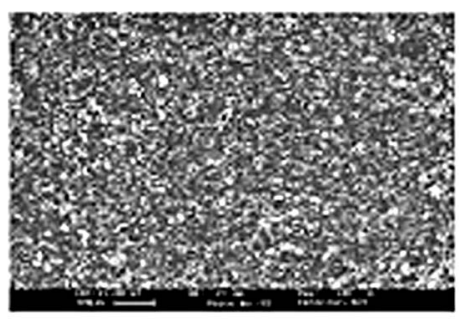

(b)

SEM micrographs of (a) 30PS/70PMMA (b) 30PS/70PMMA/CdS

Figure 5. SEM micrographs for studied PS/CdS, PMMA/CdS \& PS/PMMA/CdS samples. 


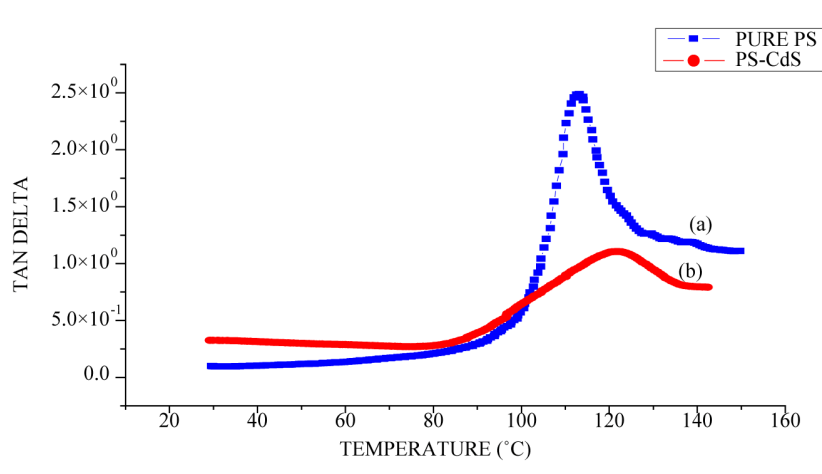

(a)

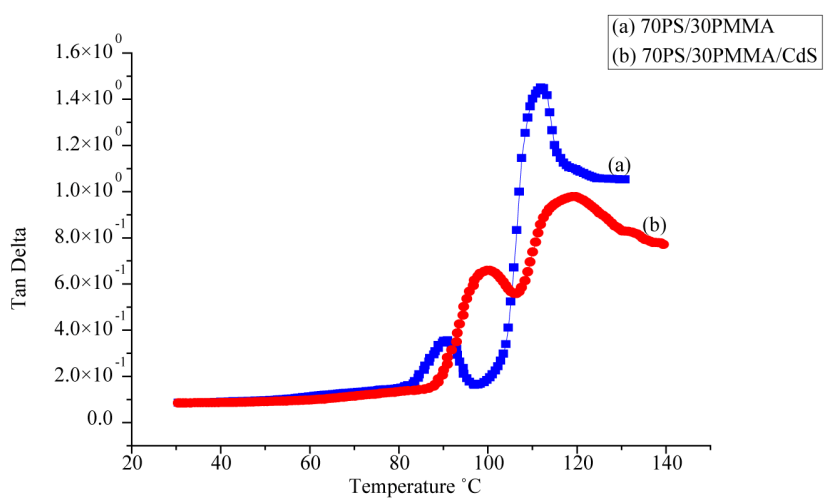

(c)

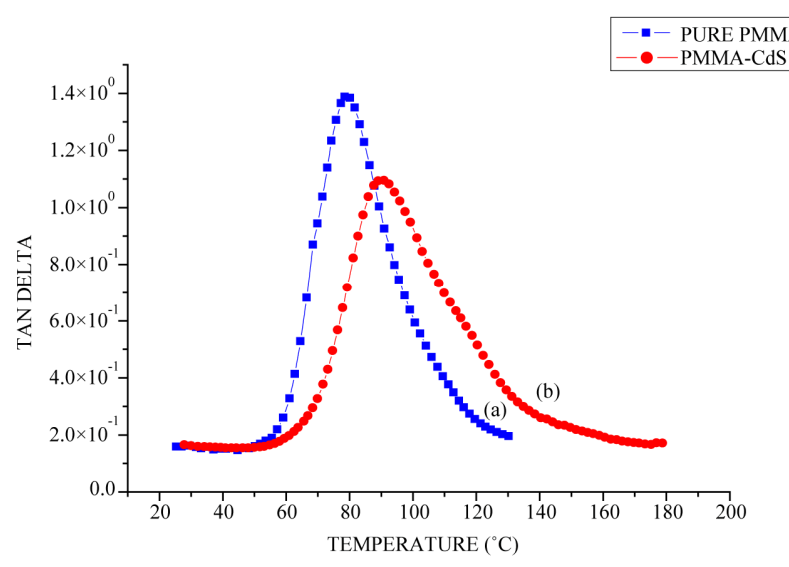

(b)

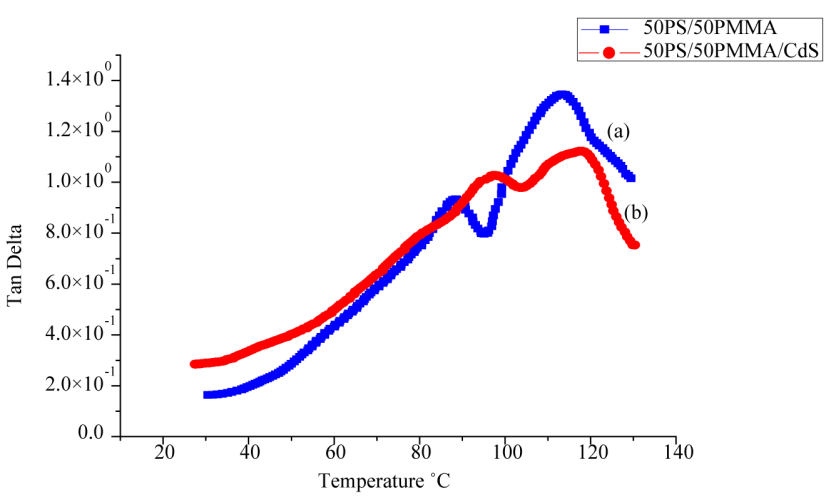

(d)

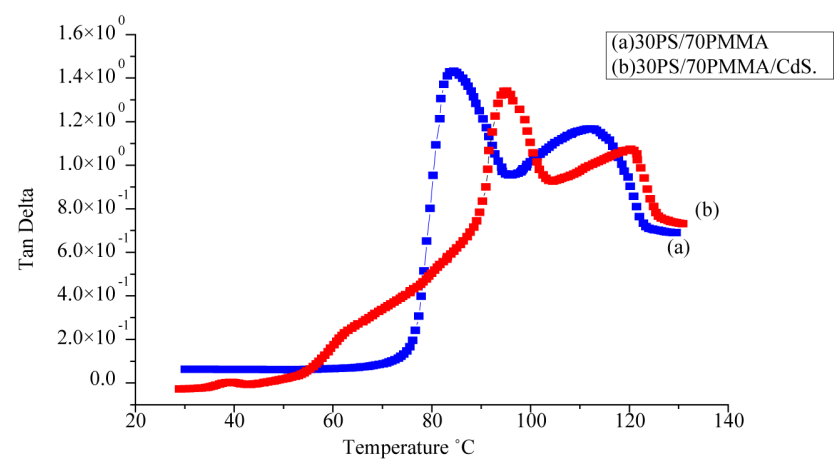

(e)

Figure 6. ((a), (b)) Variation of Tan $\delta$ with temperature for PS \& PS/CdS and PMMA \& PMMA/CdS respectively; ((c)-(e)) Variation of Tan $\delta$ with temperature of PS/PMMA and PS/PMMA/CdS systems respectively.

Table 1. Glass transition temperatures of PS/PMMA \& their CdS nanocomposites.

\begin{tabular}{|c|c|c|c|c|c|}
\hline \multirow{2}{*}{ Samples } & \multicolumn{2}{|c|}{$\operatorname{Tan} \delta$ peaks } & \multirow{2}{*}{ Samples } & \multicolumn{2}{|c|}{$\operatorname{Tan} \delta$ peaks } \\
\hline & $\mathbf{T}_{\mathrm{g}}-\mathrm{I}\left({ }^{\circ} \mathrm{C}\right)$ & $\mathbf{T}_{\mathrm{g}}-\mathrm{II}\left({ }^{\circ} \mathrm{C}\right)$ & & $\mathbf{T}_{\mathrm{g}}-\mathrm{I}\left({ }^{\circ} \mathrm{C}\right)$ & $\mathbf{T}_{\mathrm{g}}-\mathrm{II}\left({ }^{\circ} \mathrm{C}\right)$ \\
\hline Pure PS & \multicolumn{2}{|c|}{113.1} & PS/CdS & \multicolumn{2}{|c|}{121.9} \\
\hline 70PS/30PMМA & 90.57 & 112.3 & 70PS/30PMMA/CdS & 100.19 & 119.2 \\
\hline 50PS/50PMMA & 88.45 & 113.8 & 50PS/50PMMA/CdS & 97.57 & 118.18 \\
\hline 30PS/70PMMA & 84.16 & 112.7 & 30PS/70PMMA/CdS & 94.32 & 119.47 \\
\hline Pure PMMA & \multicolumn{2}{|c|}{79.3} & PMMA/CdS & \multicolumn{2}{|c|}{89.7} \\
\hline
\end{tabular}


Table 2. Thermal conductivities of PS/PMMA/CdS nanocomposites.

\begin{tabular}{cccc}
\hline Pure \& Blend samples & Thermal conductivity $(\mathrm{W} / \mathbf{m K})$ & Nanocomposite samples & Thermal conductivity (W/mK) \\
\hline Pure PS & 0.149 & PS/CdS & 0.202 \\
70PS/30PMMA & 0.159 & 70PS/30PMMA/CdS & 0.211 \\
50PS/50PMMA & 0.164 & 50PS/50PMMA/CdS & 0.218 \\
30PS/70PMMA & 0.171 & 30PS/70PMMA/CdS & 0.228 \\
Pure PMMA & 0.179 & PMMA/CdS & 0.234 \\
\hline
\end{tabular}

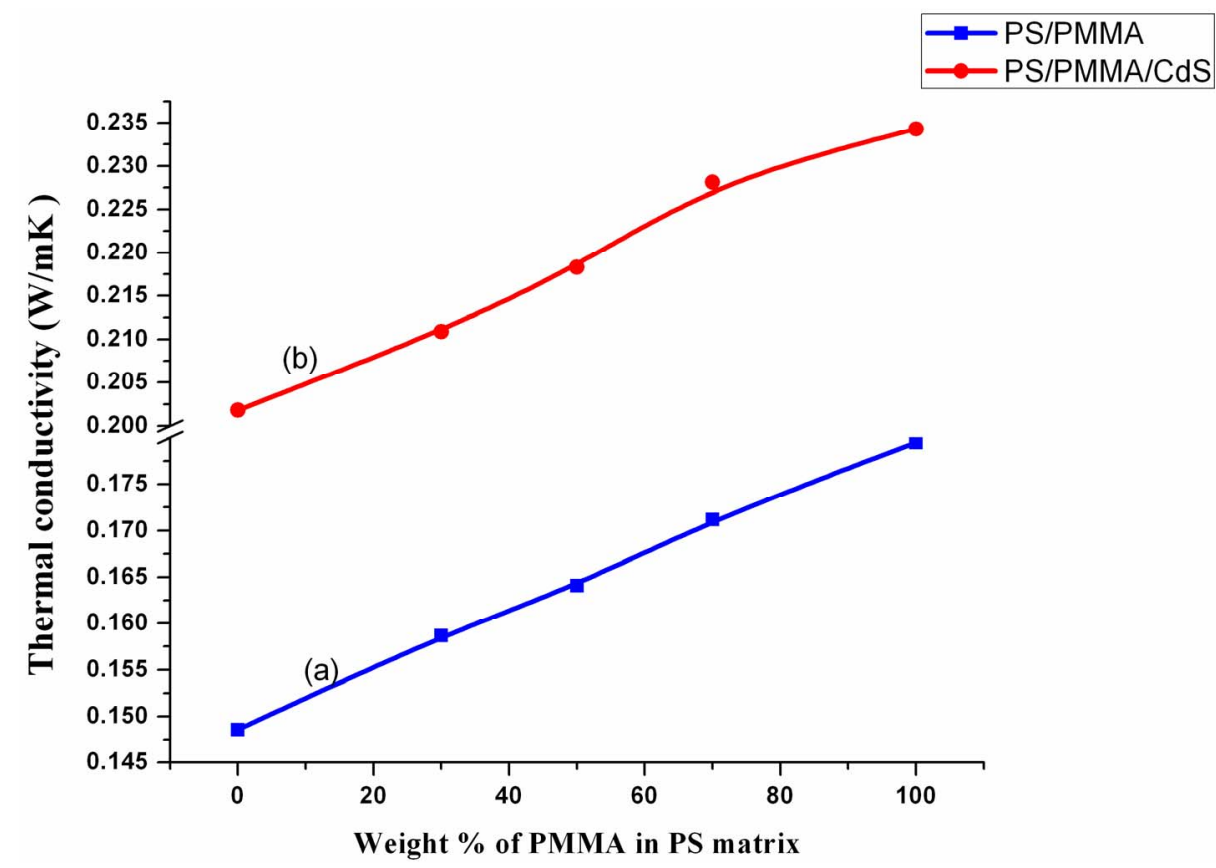

Figure 7. Variation of thermal conductivity with weight\% of PMMA in PS/PMMA/CdS nanocomposites.

the estimation of thermal properties.

Generally, the internal interfaces impede the flow of heat due to phonon scattering. At interface or grain boundary between similar materials, the interface disorder scatters phonons, while the differences in elastic properties and densities of vibrational states affect the transfer of vibrational energy across the interfaces between dissimilar materials. As a result, the nanocomposite structures exhibiting high interfaces densities depict low thermal conductivity.

\subsection{Tensile Stress-Strain Analysis}

Figure 8(a) show the tensile stress-strain characteristics of PS/CdS nanocomposite samples. It is observed that PS matrix becomes more brittle due to the dispersion of $\mathrm{CdS}$ nanoparticles. However, Young's modulus of the PS matrix remains almost constant but fracture energy of PS matrix trims down from $0.029 \mathrm{~J}$ to $0.018 \mathrm{~J}$. The ultimate tensile strength and fracture strain are observed $8.84 \mathrm{MPa}$ and $0.83 \%$, respectively for this nanocomposite.

It is seemed due to the fact that the dispersion of $\mathrm{CdS}$ nanoparticles disturbs the molecular chain network of PS matrix. This interruption causes decrease in covalent bond molecular network and increase in van der waal interaction within the nano-CdS dispersed PS matrix. This inturn induces more brittleness in the PS matrix and causing loss in strength and fracture energy of the system.

Figure 8(b) shows the tensile behavior of PMMA/CdS nanocomposite sample. The tensile properties of this nanocomposite sample are also significantly improved due to presence of CdS nanoparticles. The percentage increase observed in Young's modulus and ultimate tensile strength is $40.65 \%$ and $48.75 \%$, respectively. The fracture energy and fracture strain of this system have also been found to increase i.e. $0.24 \mathrm{~J}$ and $5.9 \%$, respectively. The improvement in tensile properties of PMMA/CdS nanocomposite is again due to the formation of compact structure. Hence dispersion of $\mathrm{CdS}$ nanoparticles provides strength to PMMA matrix. Figures 8(c)-(e) show the tensile stress strain behaviour of 70PS/30PMMA, 50PS/50PMMA and 30PS/70PMMA along with their CdS nanocomposites, respectively. It is observed that the interaction between polymer blend's molecules and nanofiller significantly 


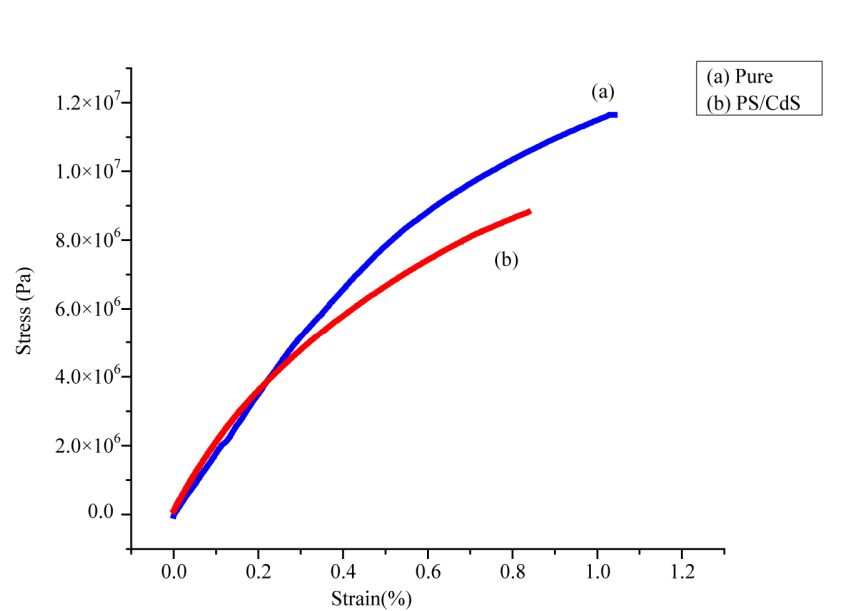

(a)

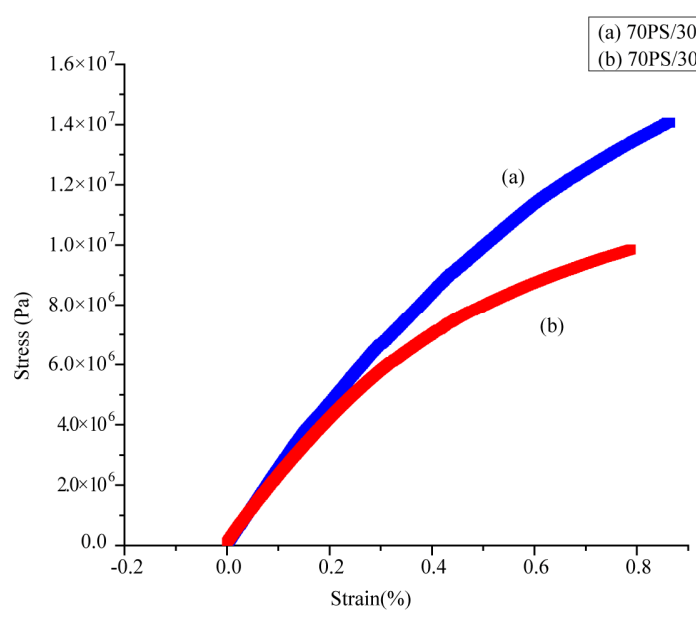

(c)

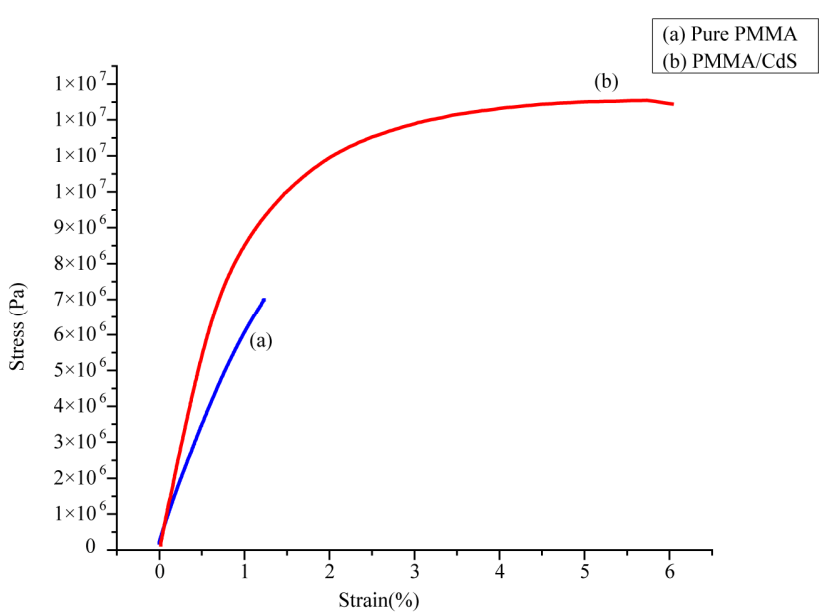

(b)

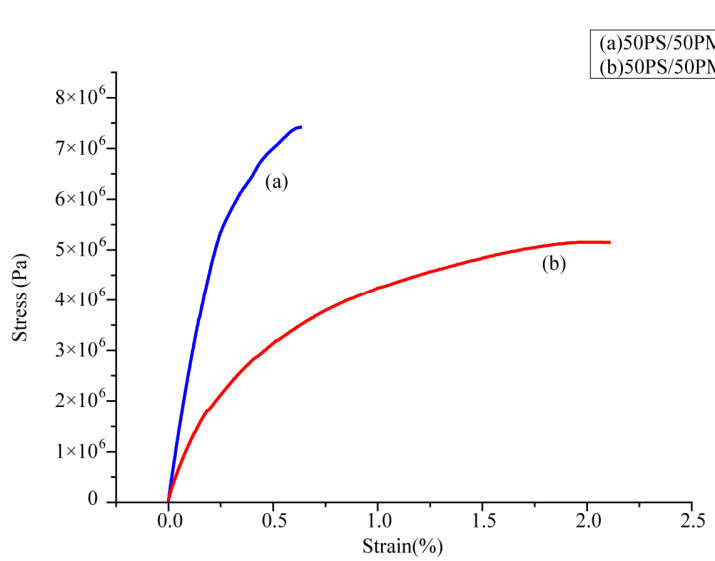

(d)

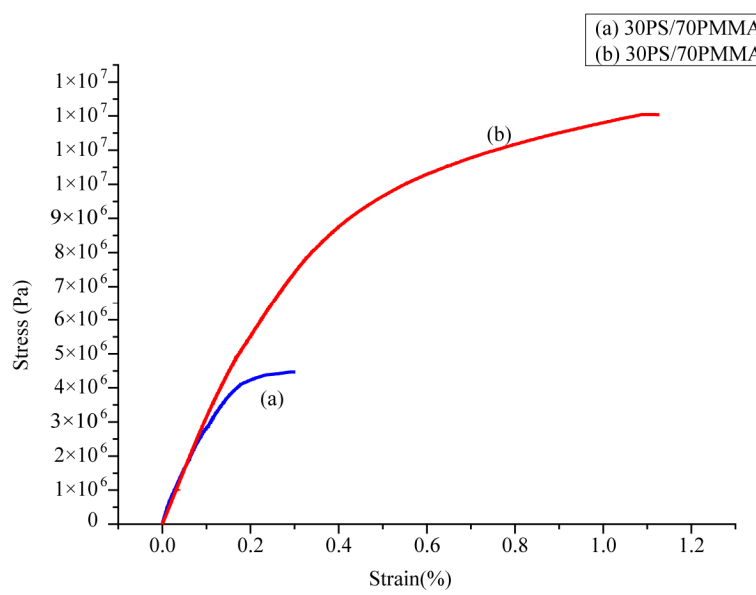

(e)

Figure 8. ((a), (b)) Stress-Strain behavior of PS \& PS/CdS and PMMA \& PMMA/CdS; ((c)-(e)) Stress-Strain behavior of PS/PMMA \& PS/PMMA/CdS samples.

alter the tensile properties of PS/PMMA blend matrix. Figure 8(c) for 70PS/30PMMA blend and its CdS nanocomposite further suggest that rich portion of blend matrix $(70 \%)$ i.e. PS phase becomes more brittle on inter- acting with dispersed CdS nanoparticles. This inturn reduces Young's modulus, ultimate strength, fracture energy and fracture strain of this nanocomposite system.

Figure 8(d) curves (a) and (b) present the tensile prop- 
Table 3. Tensile properties of PS/PMMA/CdS nanocomposite samples.

\begin{tabular}{|c|c|c|c|c|c|c|c|c|c|c|}
\hline \multirow[b]{2}{*}{ Samples } & \multicolumn{2}{|c|}{ PS } & \multicolumn{2}{|c|}{ 70PS/30PMМА } & \multicolumn{2}{|c|}{ 50PS/50PMMA } & \multicolumn{2}{|c|}{ 30PS/70PMMA } & \multicolumn{2}{|c|}{ PMMA } \\
\hline & $\begin{array}{c}\text { Without } \\
\text { CdS }\end{array}$ & With CdS & $\begin{array}{l}\text { Without } \\
\text { CdS }\end{array}$ & With CdS & $\begin{array}{l}\text { Without } \\
\text { CdS }\end{array}$ & With CdS & $\begin{array}{c}\text { Without } \\
\text { CdS }\end{array}$ & With CdS & $\begin{array}{l}\text { Without } \\
\text { CdS }\end{array}$ & With CdS \\
\hline Young modulus (GPa) & 16.64 & 15.41 & 22.05 & 19.05 & 23.29 & 9.63 & 27.92 & 29.17 & 6.42 & 9.03 \\
\hline $\begin{array}{l}\text { Ultimate strength } \\
\text { (MPa) }\end{array}$ & 11.64 & 8.84 & 14.01 & 11.20 & 7.39 & 5.14 & 4.45 & 12.03 & 7.63 & 11.35 \\
\hline Fracture energy $(\mathrm{J})$ & 0.029 & 0.018 & 0.028 & 0.019 & 0.012 & 0.033 & 0.002 & 0.039 & 0.018 & 0.24 \\
\hline Fracture strain (\%) & 1.034 & 0.83 & 0.86 & 0.78 & 0.62 & 2.09 & 0.29 & 1.12 & 1.22 & 5.94 \\
\hline
\end{tabular}

erties of 50PS/50PMMA blend and 50PS/50PMMA/CdS nanocomposite. In this nanocomposite system both the phases of PS and PMMA are defined in equal proportion with higher degree of immiscibility. Dispersion of $\mathrm{CdS}$ nanoparticles induces increase in brittle nature of PS phase and increase in ductility of PMMA phase. Thus the overall effect appears as decrement in Young's modulus and ultimate tensile strength and increment in fracture energy and fracture strain properties of this nanocomposite system.

Figure 8(e) shows the tensile stress strain behavior of 30PS/70PMMA/CdS nanocomposite along with 30PS/ 70PMMA blend. In this case also, the tensile properties have been observed significantly influenced from dominant PMMA/CdS phase. This nanocomposite system present such a compatible system for which along with Young's modulus and ultimate strength, fracture energy and fracture strain are also observed enhanced. Table 3 presents the systematic results evaluated from tensile property of PS/PMMA/CdS nanocomposite system.

The results obtained for PS/PMMA/CdS nanocomposites are principally explained on the basis of compactness and interaction between nanoparticles \& polymeric phases of the nanocomposite systems. When CdS nanoparticles are dispersed into the polymer matrix, they tend to settle down at the voids \& interface positions and act as cross-linking centers between the polymer molecules. These CdS nanoparticles behave as a surfactant, reducing the interfacial energy between the two polymer phases $[1,2]$. Since PS/PMMA blend systems are immiscible, the incorporation of the $\mathrm{CdS}$ nanoparticles with different composition dependent morphological blend structures present tailored tensile properties. Bonding between nanoparticles and polymer matrix leads good adhesion between matrix and filler [8] leading to higher density of nanocomposite structure. In this way compactness of composite is directly related to their respective prominent elastic properties.

\section{Conclusion}

This study showed that the thermal and mechanical properties of PS/PMMA immiscible polymer blends are sig- nificantly influenced by the dispersion of CdS nanofiller particles, but the effect is composition dependent. The glass transition temperature $\left(\mathrm{T}_{\mathrm{g}}\right)$ of the respective polymeric phases embedded with nanofiller $\mathrm{CdS}$ have been found to be shifted towards higher temperature side. The thermal conductivity of $\mathrm{CdS}$ nanocomposites is found to increase because of the formation of bridged polymer network through higher thermal conducting element i.e. $\mathrm{CdS}$ nanoparticles, leading to the increase in the phonon-phonon interaction within the nanocomposite matrix. It is found that PS matrix become more brittle due to dispersion of CdS nanoparticles where as compactness of PMMA matrix enhances. An increment in fracture energy and fracture strain of 50PS/50PMMA/CdS nanocomposite is also observed.

\section{REFERENCES}

[1] V. V. Ginzburg, F. Qiu, M. Paniconi, G. Peng, D. Jasnow and A. C. Balazs, "Simulation of Hard Particles in a Phase-Separating Binary Mixture," Physical Review Letters, Vol. 82, No. 20, 1999, pp. 4026-4029. doi:10.1103/PhysRevLett.82.4026

[2] Y. Tang and T. J. Ma, "Controlling Structural Organization of Binary Phase-Separating Fluids through Mobile Particles," Chemical Physics, Vol. 116, No. 17, 2002, pp. 7719-7723.

[3] M. Laradji and G. J. MacNevin, "Phase Separation Dynamics in Binary Fluids Containing Quenched or Mobile Filler Particles," Chemical Physics, Vol. 119, No. 4, 2003, pp. 2275-2283.

[4] H. Tanaka, A. J. Lovinger and D. D. Davis, "Pattern Evolution Caused by Dynamic Coupling between Wetting and Phase Separation in Binary Liquid Mixture Containing Glass Particles," Physical Review Letters, Vol. 72, No. 16, 1994, pp. 2581-2584. doi:10.1103/PhysRevLett.72.2581

[5] C. Minelli, I. Geissbuehler, R. Eckert, H. Vogel, H. Heinzelmann and M. Liley, "Organization of Nanoscale Objects via Polymer Dimixing," Colloid and Polymer Science, Vol. 282, No. 11, 2004, pp. 1274-1278. doi:10.1007/s00396-004-1070-y

[6] H. J. Chung, A. Taubert, R. D. Deshmukh and R. J. Composto, "Mobile Nanoparticles and Their Effect on Phase Separation Dynamics in Thin-Film Polymer Blends," 
Europhysics Letters, Vol. 68, No. 2, 2004, p. 219. doi:10.1209/epl/i2004-10242-2

[7] J. Bashnagel and K. Binder, "On the Influence of Hard Walls on Structural Properties in Polymer Glass Simulation," Macromolecules, Vol. 28, No. 20, 1995, pp. 68086818. doi: $10.1021 / \mathrm{ma} 00124 \mathrm{a} 016$

[8] J. Kraus, P. Müller-Buschbaum, T. Kuhlmann, D. W. Schubert and M. Stamm, "Confinement Effects on the Chain Conformation in Thin Polymer Films," Europhysics Letters, Vol. 49, No. 2, 2000, p. 210. doi:10.1209/epl/i2000-00135-4

[9] M. Vacatello, "Monte Carlo Simulations of Polymer Melts Filled with Solid Nanoparticles," Macromolecules, Vol. 34, No. 6, 2001, pp. 1946-1952.

[10] L. Wang, Y. S. Liu, X. Jiang, D. H. Qin and Y. Cao, "Enhancement of Photovoltaic Characteristics Using a Suitable Solvent in Hybrid Polymer/Multiarmed CdS Nanorods Solar Cells," Journal of Physical Chemistry C, Vol. 111, No. 26, 2007, pp. 9538-9542. doi:10.1021/ip0715777

[11] S. Kundu and H. Liang, "Photochemical Synthesis of Electrically Conductive CdS Nanowires on DNA Scaffolds," Advanced Materials, Vol. 20, No. 4, 2008, PP. 826-831. doi:10.1002/adma.200702162

[12] Y. J. Hsu, S. Y. Lu and Y. F. Lin, "Formation of Polycyanoacrylate-Silica Nanocomposites by Chemical Vapor Deposition of Cyanoacrylates on Aerogels," Chemistry of Materials, Vol. 20, No. 9, 2008, pp. 2854-2856. doi:10.1021/cm7030703

[13] Y. F. Lin, J. Song, Y. Ding, S. Y. Lu and L. Wang, "Fabrication and Light-Transmission Properties of Monolayer Square Symmetric Colloidal Crystals via Controlled Convective Self-assembly on 1D Grooves," Advanced Materials, Vol. 20, No. 1, 2008, pp. 123-128. doi:10.1002/adma.200701175

[14] J. C. Lee, W. Lee, S. H. Han, T. G. Kim and Y. M. Sung, "Synthesis of Hybrid Solar Cells Using CdS Nanowire Array Grown on Conductive Glass Substrates," Electrochemistry Communications, Vol. 11, No. 1, 2009, pp. 231234. doi:10.1016/j.elecom.2008.11.021

[15] C. Li, J. Zhu, Q. Li, S. Chen and Y. R. Wang, "Controllable Synthesis of Functionalized CdS Nanocrystals and CdS/PMMA Nanocomposite Hybrids," European Polymer Journal, Vol. 43, No. 11, 2007, pp. 4593-4601. doi:10.1016/j.eurpolymi.2007.08.008

[16] M. Z. Rong, M. Q. Zhang, H. C. Liang and H. M. Zeng, "Surface Modification and Particles Size Distribution Control in Nano-CdS/Polystyrene Composite Film," Chemical Physics, Vol. 286, No. 2-3, 2003, pp. 267-276. doi:10.1016/S0301-0104(02)00928-X

[17] J. H. Zeng, J. Yang, Y. Zhu, Y. F. Liu, Y. T. Qian and H. G. Zheng, "Nanocomposite of CdS Particles in Polymer Rodsfabricated by a Novel Hydrothermal Polymerization and Simultaneous Sulfidation Technique," Chemical Communications, 2001, pp. 1332-1333.

[18] D. Schlemmer, E. R. de Oliveira and M. J. Araújo Sales, "Polystyrene/Thermoplastic Starch Blends with Different
Plasticizers," Journal of Thermal Analysis and Calorimetry, Vol. 87, No. 3, 2007, pp. 635-638.

[19] J. K. Chen, S. W. Kuo, H. C. Kao and F. C. Chang, "Thermal Properties, Specific Interactions, and Surface Energies of PMMA Terpolymers Having High Glass Transition Temperatures and Low Moisture Absorptions," Polymer, Vol. 46, No. 7, 2005, pp. 2354-2364. doi:10.1016/j.polymer.2005.01.046

[20] K. S. Rathore, D. Patidar, Y. Janu, N. S. Saxena, K. B. Sharma and T. P. Sharma, "Structural and Optical Characterization of Chemically Synthesized $\mathrm{ZnS}$ Nanoparticles," Chalcogenide Letters, Vol. 5, No. 6, 2008, pp. 105110.

[21] S. Gupta, D. Patidar, N. S. Saxena, K. B. Sharma and T. P. Sharma, "Electrical Study of Cu-CdS and Zn-CdS Schottky Junction," Advanced Materials, Vol. 2, No. 4, 2008, pp. 205.

[22] M. Dixit, S. Gupta, V. Mathur, K. S. Rathore, K. Sharma and N. S. Saxena, "Study of Glass Transition Temperature of PMMA and CdS-PMMA Composite," Chalcogenide Letters, Vol. 6, No. 3, 2009, pp. 131-136.

[23] S. E. Gustafsson, "Transient Plane Source Techniques for Thermal Conductivity and Thermal Diffusivity Measurements of Solid Materials," Review of Scientific Instruments, Vol. 62, No. 3, 1991, p. 797.

[24] K. Menard, "Dynamic Mechanical Analysis, A Practical Introduction," 1999.

[25] S. W. Choi, J. H. Yoon, M. J. An, W. S. Chae, H. M. Cho, M. G. Choi and Y. R. Kim. "Organic Nanotube Induced by Photocorrosion of CdS Nanorod," Bulletin of Korean Chemical Society, Vol. 25, No. 7, 2004, pp. 983-985.

[26] X. F. Lu, H. Mao, W. J. Zhang and C. Wang, "Synthesis and Characterization of CdS Nanoparticles in Polystyrene Microfibers," Materials Letters, Vol. 61, No. 11-12, 2007, pp. 2288-2291. doi:10.1016/j.matlet.2006.08.070

[27] B. J. Ash, L. S. Schadler and R. W. Siegel, "Glass Transition Behavior of Alumina/Polymethylmethacrylate Nanocomposites," Materials Letters, Vol. 55, No. 1-2, 2002, pp. 83-87. doi:10.1016/S0167-577X(01)00626-7

[28] F. Mammeri, E. Le Bourhis, L. Rozesa and C. Sanchez, "Mechanical Properties of Hybrid Organic-Inorganic Materials," Journal of Materials Chemistry, Vol. 15, No. 35-36, 2005, pp. 3787-3811. doi:10.1039/b507309j

[29] Z. Gao, W. Xie, J. M. Hwu, L. Wells and W.-P. Pan, "The Characterization of Organic Modified Montmorillonite and Its Filled PMMA Nanocomposite," Journal of Thermal Analysis and Calorimetry, Vol. 64, 2001, pp. 467-475. doi:10.1023/A:1011514110413

[30] A. P. Whittington, S. T. Nguyen and J.-H. Kim, "Thermal Behavior of Polystyrene-Silica Nanocomposites," Nanoscape, Vol. 6, No. 1, 2009, pp. 26-29.

[31] Q. Zhao and E. T. Samulski, "A Comparative Study of Poly(methyl methacrylate) and Polystyrene/Clay Nanocomposites Prepared in Supercritical Carbon Dioxide,' Polymer, Vol. 47, No. 2, 2006, pp. 663-671. doi:10.1016/j.polymer.2005.11.079 